\title{
Stérilisation - Aperçu des conditions légales
}

\author{
H. Schmid
}

\section{Loi sur la stérilisation}

Les recommandations du 12 juin 2001 de l'Académie Suisse des Sciences Médicales concernant la stérilisation de personnes mentalement déficientes (complément aux directives de 1981) prévoient que «dans certains cas, il peut y avoir des motifs de stérilisation chirurgicale de personnes incapables de discernement avec une déficience mentale. Au vu du vide juridique actuel, dans de tels cas, l'on ne pratiquera pas la stérilisation. [...] Il est du ressort des instances législatives de formuler des conditions-cadres en vue d'autoriser de telles interventions.»

Donnant suite à une initiative parlementaire von Felten, la loi fédérale du 17 décembre 2004 sur les conditions et la procédure régissant la stérilisation de personnes [ci-après: Lstér]; RS

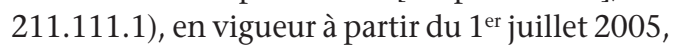
règle en particulier les conditions auxquelles une stérilisation est autorisée à des fins contraceptives (art. 1 Lstér). La stérilisation de personnes durablement incapables de discernement (art. 7 Lstér) constitue l'objet principal de cette loi.

\section{Définition légale de la stérilisation}

La loi sur la stérilisation définit la stérilisation sur les plans médical et légal:

- L'art. 2, al. 3, Lstér prévoit une réserve - qui va de soi - en faveur des médecins, à savoir que seul un médecin peut pratiquer des stérilisations. La stérilisation constitue une intervention médicale qui consiste à supprimer de manière permanente les facultés reproductrices d'une personne (art. 2, al. 1, Lstér), au moyen d'une ligature des trompes ou d'une vasectomie. «De manière permanente» ne signifie pas «de façon définitive»; actuellement, grâce à la microchirurgie, la stérilisation est réversible dans de nombreux cas. Il y a lieu - notamment en ce qui concerne les personnes durablement incapables de discernement - de toujours choisir le mode d'opération dont la probabilité de réversibilité est la plus élevée (cf. art. 7, al. 2, let. f, Lstér).

Dr iur. Hermann Schmid, avocat Office fédéral de la justice

Division principale du droit privé Division des projets de législation CH-3003 Berne

E-mail:

hermann.schmid@bj.admin.ch trices de la personne (art. 2, al. 2, Lstér). Si l'intervention a été pratiquée sur une personne incapable de discernement, le médecin a l'obligation de l'annoncer dans les dix jours à l'autorité tutélaire de surveillance (art. 10, al. 1, Lstér).

\section{Stérilisation selon les personnes}

\section{Majeurs}

Personnes capables de discernement

Une stérilisation ne peut être pratiquée sur une personne âgée de plus de 18 ans et capable de discernement que si elle a été informée de manière détaillée sur l'intervention et que si elle y a consenti librement. L'obligation de consentir par écrit (art. 5, al. 1, Lstér) a pour but de garantir que la personne décide en toute connaissance de cause.

Le médecin qui pratique la stérilisation doit consigner dans le dossier médical les éléments qui ont fondé son appréciation de la capacité de discernement de la personne concernée (art. 5, al. 2, Lstér). Cette règle veut empêcher une évaluation insuffisante de la capacité de discernement de la personne concernée. Le médecin qui pratique la stérilisation peut se baser sur des constatations établies par le médecin traitant.

Personnes capables de discernement et interdites L'art. 6 Lstér fixe les conditions auxquelles la stérilisation peut être pratiquée sur une personne âgée de 18 ans capable de discernement et interdite. Une personne âgée de 16 ou 17 ans, donc mineure, est pourvue d'un tuteur si elle n'est pas sous autorité parentale (art. 368, al. 1, CC); toutefois, l'institution d'une tutelle sur un mineur ne constitue pas une interdiction au sens d'une suppression totale de l'exercice des droits civils par l'autorité. La tutelle instituée en raison de la minorité (et par défaut d'autorité parentale) n'exige pas l'interdiction préalable de la personne. Ainsi, les personnes âgées de 16 ou 17 ans et placées sous tutelle sont protégées par l'art. 3, $1^{\text {re }}$ phrase, Lstér, qui interdit la stérilisation d'une personne âgée de moins de 18 ans.

L'art. 6, al. 1, $1^{\text {re }}$ phrase, Lstér exige le consentement libre, éclairé et écrit de la personne concernée. Cette condition est déjà prévue par 
l'art. 5, al. 1, Lstér pour la stérilisation d'une personne capable de discernement. C'est pourquoi la règle de l'art. 6, al. 2, let. a, Lstér, selon laquelle les éléments qui ont fondé l'appréciation de la capacité de discernement doivent être consignés dans le dossier médical, correspond à l'art. 5, al. 2, Lstér.

Les majeurs qui, bien que capables de discernement, sont placés sous tutelle (art. 369 à 372 CC), nécessitent une protection spéciale, car ils ne sont souvent plus en mesure de défendre leurs intérêts en raison de l'état de faiblesse dans lequel ils se trouvent. En conséquence, avant de pratiquer la stérilisation, le médecin doit, d'une part, requérir le consentement du représentant légal (art. 6, al. 1, 2e phrase, Lstér), et, d'autre part, demander l'approbation de l'autorité de surveillance (art. 6, al. 2, let. b, Lstér). Ces deux consentements constituent une protection supplémentaire pour la personne interdite.

La capacité de discernement de la personne interdite doit toujours - et non seulement en cas de doute - être évaluée par un autre médecin que celui qui pratique la stérilisation (art. 6, al. 2, Lstér). C'est pourquoi l'autorité tutélaire de surveillance doit demander un second avis médical (art. 6, al. 3, 1re phrase, Lstér). Si nécessaire, elle ordonne une expertise psychiatrique (art. 6, al. $3,2^{\mathrm{e}}$ phrase, Lstér). Une telle expertise constitue une charge pour la personne qui doit s'y soumettre; dès lors, elle ne doit être ordonnée que si, malgré les informations complémentaires qui ont été fournies, il subsiste des doutes importants sur la capacité de discernement. Dans la plupart des cas, le second avis médical suffira pour dissiper les incertitudes de l'autorité tutélaire de surveillance. Il y a lieu de noter qu'il est interdit de demander une expertise psychiatrique pour la seule raison que la personne est sous tutelle.

\section{Personnes passagèrement incapables} de discernement

En présence d'une incapacité de discernement probablement passagère, la stérilisation ne doit pas être interdite (art. 4, Lstér), car l'intervention ne doit pas avoir pour conséquence de supprimer la faculté reproductrice de la personne concernée sur une longue durée.

Si une expertise médicale permet d'établir avec certitude que la personne recouvrera une capacité de discernement qui sera limitée, ce sont les règles applicables aux personnes durablement incapables de discernement qui s'appliquent (voir ci-après).

\section{Mineurs}

Le législateur est d'avis que les jeunes personnes manquent souvent de la maturité nécessaire pour saisir toute la portée d'une stérilisation et pour établir un projet parental. C'est pourquoi la stérilisation d'une personne âgée de moins de 18 ans est en principe interdite (art. 3, $1^{\text {re }}$ phrase, Lstér); elle est autorisée à 18 ans révolus. La stérilisation d'une personne âgée de plus de 16 ans et durablement incapable de discernement est exceptionnellement autorisée à des conditions strictes (art. 3, 2e phrase, en rapport avec art. 7 Lstér).

\section{Personnes durablement incapables de discernement}

\section{Condition formelle}

Le médecin ne peut procéder à une stérilisation qu'avec l'autorisation de l'autorité tutélaire de surveillance (art. 7, al. 2, let. g, en rapport avec art. 8, al. 1, Lstér). Les cantons doivent, en vertu du droit fédéral, désigner l'autorité de surveillance, qui peut comprendre deux instances au maximum, soit une instance inférieure et une instance supérieure (art. 361 CC). Les cantons qui ont prévu deux instances attribueront, en règle générale, la compétence d'autoriser les stérilisations à l'instance inférieure. La détermination du for relève du droit cantonal.

\section{Conditions matérielles}

Avant de donner son autorisation, l'autorité tutélaire de surveillance doit examiner si toutes les conditions cumulatives énumérées ci-après sont réalisées (cf. le terme «et» dans l'art. 7, al. 2, let. f, Lstér):

- La conception et la naissance d'un enfant ne peuvent pas être empêchées par d'autres méthodes de contraception appropriées ou la stérilisation volontaire du partenaire capable de discernement (art. 7, al. 2, let. b, Lstér). En vertu du principe de la proportionnalité, il devrait être fait recours à des méthodes de contraception réversibles.

- La personne concernée est âgée de plus de 16 ans (art. 7, al. 1, Lstér).

- Il est établi, sur la base d'un avis médical, que la personne concernée est durablement incapable de discernement (art. 7, al. 1; art. 8, al. 2 , let. c, Lstér) et qu'elle n'a aucune chance d'acquérir cette capacité (art. 7, al. 2, let. e, Lstér); en outre, une grossesse mettrait sérieusement en danger la santé de la femme (art. 7, al. 2, let. d, Lstér). 
- Le projet de loi sur la stérilisation autorisait l'intervention seulement «dans l'intérêt de la personne concernée» (art. 7, al. 2, let. a, P Lstér). Par contre, le Parlement a opté pour une formulation plus large, qui autorise la stérilisation dans l'intérêt de la personne concernée, pour autant toutefois que toutes les circonstances aient été considérées (art. 7, al. 2, let. a, Lstér). L'élément déterminant en faveur de cette solution a été la prise en considération du fait que les parents et les frères et sœurs de la personne handicapée mentale sont concernés par une éventuelle grossesse, car celle-ci constitue indubitablement une charge supplémentaire importante pour eux. Enfin, il faut prendre en compte également les intérêts de l'enfant. C'est pourquoi le législateur s'est opposé à la solution qui ne prend pas en considération les intérêts des tiers.

Ce même projet n'autorisait la stérilisation que si la personne concernée «n'a pas manifesté d'opposition à l'intervention» (art. 7, al. 2, let. a, P Lstér). Une peur diffuse de l'intervention constituait déjà une opposition juridiquement valable. Le médecin n'était pas autorisé à prescrire à la personne concernée des médicaments pour l'aider à surmonter ses appréhensions face à l'intervention; il ne pouvait que lui accorder un entretien visant à éclaircir la situation. L'autodétermination de la personne concernée aurait été prise en considération jusqu'au dernier moment. Mais ce concept de «volonté naturelle (qui s'oppose)» de la personne incapable de discernement aurait conduit à une interdiction de fait de la stérilisation, car de nombreuses personnes ayant un handicap mental grave ont une peur disproportionnée des examens ou des actes médicaux. Toutefois, une décision négative aurait pu avoir pour la femme des conséquences encore plus dures: une grossesse, un accouchement ou un avortement ainsi que des mesures visant à empêcher des contacts sexuels, qui constituent une limitation de la liberté personnelle. Le Parlement a également rejeté cette solution.

- Toujours selon le projet, la stérilisation n'était autorisée que si une grossesse, la venue au monde d'un enfant ou la séparation inévitable d'avec l'enfant mettaient sérieusement en danger la santé physique ou psychique de la femme ou de l'homme (art. 7, al. 2, let. d, P Lstér). Mais le Parlement est parvenu à la conviction qu'il n'y avait pas de sens à interdire la stérilisation en particulier lorsque la personne concernée ne manifeste aucune émotion en raison de son handicap mental. C'est pourquoi la condition a été formulée de manière plus objective et l'indication relative à la séparation d'avec l'enfant a été conçue comme condition indépendante et rédigée de la manière suivante: «la séparation d'avec l'enfant après la naissance est inévitable parce que les responsabilités parentales ne peuvent pas être exercées» (art. 7, al. 2, let. d, Lstér).

- La stérilisation n'est autorisée que si «la conception et la naissance d'un enfant sont à prévoir» (art. 7, al. 2, let. c, Lstér). Ainsi, la stérilisation à titre préventif d'une femme inactive sexuellement en prévision d'un danger abstrait de viol n'est pas admissible, ce qui ne signifie pas qu'une personne durablement incapable de discernement devrait avoir des relations sexuelles sans être protégée pour remplir les conditions de la stérilisation. Comme aujourd'hui les homes, les communautés etc. qui accueillent des personnes ayant un handicap mental ne connaissent pratiquement plus la séparation selon le sexe, une protection en temps voulu contre une grossesse non désirée est nécessaire, car le personnel ne peut guère prévoir le début précis de relations intimes. 